D.O.I.:10.3895/S1808-04482013000400003

\title{
METODOLOGIA PARA AVALIAR A CAPACIDADE DE INOVAÇÃO TECNOLÓGICA NA PERFORMANCE DE EMPRESAS HIGH TECH
}

\section{METHODOLOGY FOR EVALUATION THE TECHNOLOGICAL INNOVATION CAPABILITIES IN PERFORMANCE OF FIRMS HIGH TECH}

\author{
Selma Regina Martins Oliveira ${ }^{1}$; Jorge Lino Alves ${ }^{2}$ \\ ${ }^{1}$ Universidade de São Paulo/USP - Brasil \\ selmaregina@webmail.uft.edu.br \\ ${ }^{2}$ INEGI, Faculdade de Engenharia/Universidade do Porto - Portugal \\ falves@fe.up.pt
}

\begin{abstract}
Resumo
Este trabalho tem por objetivo contribuir para uma política de planejamento de inovação em empresas high tech no Brasil. Para isto apresenta uma metodologia para avaliar a capacidade de inovação tecnológica na performance desta categoria de empresas. Este procedimento foi elaborado conforme as seguintes fases: Fase 1: determinação do modelo conceitual; e fase 2: verificação do modelo conceitual:. A pesquisa contou com a intervenção de especialistas. Foram utilizados complementarmente os métodos: Análise Multicriterial, Análise Multivariada; Escalagem Psicométrica e Tecnologia Neurofuzzy. Os dados foram coletados por meio de uma matriz de julgamento. Os resultados mostraram-se satisfatórios validando a proposta de metodologia. Neste cenário, esta contribuição metodológica é elevada porque estabelece prioridades na implementação de projetos inovadores, e também permite direcionar a inovação como um elemento essencial para o desenvolvimento de novos produtos. Este suporte procura ainda estabelecer uma orientação mais pragmática e eficiente em metas de longo prazo, assegurando a competitividade nacional na perspectiva de prioridades, que dependem do estado inicial da arte e das características dos projetos e políticas de inovação.
\end{abstract}

Palavras-chave: metodologia; avaliação; capacidade de inovação tecnológica; performance de empresas high tech.

\section{Introdução}

Recentemente as mudanças relevantes tornaram as fronteiras organizacionais mais fluidas e dinâmicas em resposta ao ritmo acelerado da difusão do conhecimento (GRILICHES, 1990; TEECE, 1986), da inovação e competição internacional (CHESBROUGH e ROSENBLOOM, 2002; CHRISTENSEN, 2003; DAMANPOUR, 1996). Isto inspira reconsiderar como ganhar com a inovação (TEECE et. al., 1997; TEECE, 1986; WHEELWRIGHT e CLARK, 1992). Assim as empresas inovadoras se valem de suas capacidades de apropriar do valor econômico gerado a partir 
de seus conhecimentos e inovações (GRILICHES, 1990; TEECE, 1986). Por esta via, a oferta de produtos inovadores se apresenta como um padrão de qualidade na disputa por inadiáveis demandas.

Dá a crer que as empresas que conseguem disponibilizar seus produtos aos clientes com maior requinte de eficiência e rapidez estarão provavelmente em melhor posição para criar uma vantagem competitiva sustentável (PRAHALAD e HAMEL, 1990; CALANTON et al, 1995) à luz do conhecimento e da inovação (TEECE et al, 1997; NELSON e WINTER, 1982). Nesta dicotomia, a eficiência técnica se apresenta como parâmetro das capacidades de desenvolvimento de produtos inovadores, que se traduz em uma das mais notáveis lógicas de potencializar e favorecer vantagem competitiva (WHEELWRIGHT e CLARK, 1992; BROWN e EISENHARDT, 1995). Presume-se que um dos principais desafios é desenvolver produtos em ambientes de alta complexidade. Respostas vêm sendo dadas a esses desafios à luz de potencialidades técnicas igualmente inovadoras, maior agilidade, produtividade e alta qualidade por parte das empresas (WHEELWRIGHT e CLARK, 1992). Desenvolver produtos não é um fenômeno recente, mas de reconstrução à sua época, apresenta experiências bem sucedidas e outras fracassadas. E qualquer tentativa de encorajar uma reconstrução e uma interpretação remete antes de tudo, uma análise apropriada das dificuldades e particularidades do desenvolvimento de produtos em cadeia, das "semânticas contrapostas em razão da diversidade de características que os sistemas possuem denominações, estruturas, metodologias e organização. Seja como for, o desenvolvimento de produtos se apresenta como uma cadeia complexa de eventos e decisões, a qual pode se romper em qualquer elo mais fraco: alguns projetos se perderam em função de previsões irrealistas ou ausência de seu verdadeiro papel na agenda, ou outras motivações que de alguma forma, seguiram lógicas com muitos acidentes de percurso ou um erro no detalhe.

Neste espectro a inovação de novos produtos tem sido determinante para que as empresas sejam bem sucedidas, em particular as indústrias de alta tecnologia. A inovação tecnológica é um processo dinâmico, talvez o mais dinâmica de toda a atividade industrial. Isto requer um esforço combinado das funções organizacionais e recursos limitados. As atividades de inovação são complexas e arriscadas. Por isso, difíceis de avaliar com precisão (AFUAH, 1998; GARCÍA-MUIN e PEZ NAVAS-LO, 2007; BELLMAN e ZADEH; 1970). Avaliar depende de um juízo subjetivo, experiência anterior, conhecimento e informação. Todos esses elementos são difíceis de definir e interpretar com precisão. Assim sendo, é elementar avaliar a capacidade de inovação tecnológica das empresas. Seja como for, a literatura mostra avanços significativos. Yam et al (2004) realizaram uma pesquisa empírica sobre inovação tecnológica para empresas de manufatura na região de Pequim (GUAN et al, 2006; GUAN e MA, 2003) e os resultados apresentaram uma ou outra inconsistência. Recentemente em pesquisa realizada por Lau, Yam e Tang (2008) e Wang, Lu e 
Chen (2008) mostrou o impacto da inovação tecnológica na performance da inovação e a capacidade da inovação tecnológica da firma em condições de incerteza. $\mathrm{O}$ estudo mostrou o quanto cada capacidade de inovação tecnológica representa para a função negócios de uma organização (capacidade de $\mathrm{P}$ \& D, manufatura e capacidade de marketing) ou um negócio multifuncional(capacidade de aprendizagem, capacidade de organização, capacidade de planejamento estratégico, capacidade de alocação de recursos). Porém, nenhum dos referidos estudos evidenciaram a determinação da taxa ótima da capacidade de inovação tecnológica na performance das empresas.

Este trabalho tem por objetivo contribuir para uma política de planejamento de inovação em empresas high tech no Brasil. Para isto apresenta uma metodologia para avaliar a capacidade de inovação tecnológica na performance desta categoria de empresas à luz do desenvolvimento de novos produtos. Este procedimento foi elaborado conforme as seguintes fases e etapas: Fase 1: determinação do modelo conceitual; e fase 2: verificação do modelo conceitual: Etapa (i) Modelagem das capacidades de inovação tecnológica na performance das empresas, em duas subetapas: (i) identificação; e (ii) avaliação das capacidades de inovação tecnológica na performance das empresas. Etapa (ii): Determinação das correlações entre as capacidades de inovação e as dimensões de performance das empresas. Etapa (iii) Priorização das capacidades de inovação tecnológica das empresas em relação à performance de inovação tecnológica das empresas. Etapa (iv) Modelagem da taxa de efetividade (taxa ótima) das capacidades de inovação tecnológica para a performance das empresas. Este trabalho está estruturado conforme as seguintes seções: definição do modelo conceitual; verificação do modelo conceitual e resultados; por fim, palavras finais e lições aprendidas.

\section{Modelo conceitual: constructos e hipóteses}

Esta seção examina o modelo conceitual (Figura 1) e apresenta as hipóteses. 


$\begin{array}{ll}\text { Variáveis independentes } & \text { Variáveis dependentes } \\ \text { Dimensões da Capacidade de Inovação Tecnológica da } & \text { Performance da } \\ \text { Firma } & \text { Firma: } \\ \text { D1: Capacidade de P\&D (Guan and Ma, 2003; } & \text { P1: Impacto no } \\ \text { Burgelman } & \text { Cliente } \\ \text { et al, 2004; Yam et al, 2004) } & \text { P2: Resultado dos } \\ \text { D2: Capacidade de Decisão de Inovação (Barton, } & \text { P3: Percentual de } \\ \text { 1984) } & \text { Vendas de } \\ \text { D3: Capacidade de Marketing (Achilladelis, e } & \text { Produtos } \\ \text { Antonajis, 2001; Guan e Ma, 2003; Yam et al, 2004; } & \\ \text { Kim et al, 2005) } & \\ \text { D4: Capacidade de Manufatura (Guan and Ma, 2003; } & \\ \text { Yam et al, 2004) } & \\ \text { D5: Capacidade de Capital (Yam et al, 2004). } & \\ \text { D6: Capacidade de Alocação de Recursos (Lau, Yan e } \\ \text { Tang, 2010) } \\ \text { D7: Capacidade de Planejamento Estratégico (Lau, }\end{array}$

Variáveis dependentes: foram selecionadas as seguintes variáveis dependentes para esta pesquisa: Impacto no Cliente; Resultado dos Negócios; Percentual de Vendas de Produtos Inovadores.

Variáveis independentes: as variáveis independentes, capacidades de inovação tecnológica das empresas, foram extraídas à luz da literatura especializada. Sendo assim, foram consideradas as seguintes dimensões como variáveis independentes Dimensões da Capacidade de Inovação Tecnológica da Firma: D1: Capacidade de P\&D (GUAN e MA, 2003; BURGELMAN et al, 2004; YAM et al, 2004); D2: Capacidade de Decisão de Inovação (BARTON, 1984); D3: Capacidade de Marketing (ACHILLADELIS e ANTONAJIS, 2001; GUAN e MA, 2003; YAM et al, 2004; KIM et al, 2005); D4: Capacidade de Manufatura (GUAN e MA, 2003; YAM et al, 2004); D5: Capacidade de Capital (YAM et al, 2004); D6: Capacidade de Alocação de Recursos (LAU, YAN e TANG, 2010); D7: Capacidade de Planejamento Estratégico (LAU, YAN e TANG, 2010); D8: Capacidade de Aprendizagem (LAU, YAN e TANG, 2010); e D9: Capacidade Organizacional (LAU, YAN e TANG, 2010).

Formulação das hipóteses a partir do modelo conceitual: Hipótese 1: As capacidades de inovação tecnológica influenciam positivamente a performance das empresas High Tech. Hipótese 2: A interação entre as dimensões de capacidades de inovação tecnológica relaciona-se com a taxa de efetividade ótima da performance das empresas High Tech. 


\section{Metodologia}

O objetivo do presente estudo é avaliar a capacidade de inovação tecnológica na performance de empresas high tec no Brasil. Para isto foi desenvolvida uma pesquisa à luz da literatura para levantamento das variáveis que compõem o modelo conceitual Num segundo momento essas variáveis foram submetidas a especialistas com conhecimento sobre o objeto de pesquisa para confirmação e avaliação em relação à performance de empresas high tech. Os dados foram coletados por meio de uma matriz de julgamento em que os especialistas emitiram sua opinião sobre as capacidades de inovação tecnológica.

\section{Verificação do modelo conceitual}

Nesta seção são apresentados os procedimentos para verificação do modelo conceitual. Para resolver o problema de pesquisa e alcançar o objetivo pretendido, num primeiro momento são identificados e priorizados os subcomponentes da capacidade de inovação tecnológica das empresas high tech. Logo após esse procedimento modela-se a avaliação global da capacidade de inovação tecnológica em relação à performance das empresas high tech. Por fim, modela-se a taxa de efetividade ótima da performance das capacidades de inovação tecnológica a partir da interação entre todas as variáveis independentes.

Fase 1: Priorização dos sub-componentes da capacidade de inovação tecnológica em relação à performance global das empresas high tech

São priorizadas as dimensões de capacidade de inovação tecnológica em relação à performance das empresas high tech. Este procedimento é desenvolvido utilizando o método de escalagem psicométrica Lei dos Julgamentos Categóricos de Thurstone de 1927. Entende-se que o Método dos Julgamentos Categóricos é uma modelagem de comportamento mental que tem por objetivo explicar a estrutura das preferências dos especialistas em relação a um conjunto de estímulos. Neste trabalho, a opção pelo Método Lei dos Julgamentos Categóricos de Thurstone pode se justificar como uma ferramenta estratégica a ser testada para priorizar, por importância, as capacidades de inovação tecnológica à luz da performance das empresas high tech. Este método considera o comportamento mental para explicar a estrutura das preferências dos decisores sobre as características priorizadas. Os procedimentos para aplicação do instrumento são sistematizados nos seguintes passos: Etapa 1: Determinação das freqüências das preferências por pares de estímulos, em que $\mathrm{O}_{\mathrm{i}}$ equivale às características e $\mathrm{O}_{\mathrm{j}}$ aos especialistas - $\left.\mathrm{O}_{\mathrm{i}}\right] \mathrm{O}_{\mathrm{j}}$. Os dados aqui sistematizados foram extraídos a partir das preferências dos especialistas em relação às capacidades(mediante pesquisa de campo utilizando questionário/matriz de julgamento). As características aparecem sob forma de estímulos submetidos às categorias ordinais. Etapa 2: Determinação das frequiências das 
categorias ordinais, a partir dos dados extraídos da etapa anterior. Calcula-se a matriz [ $\pi \mathrm{ij}]$ das frequiências relativas acumuladas. Os resultados são classificados em ordem crescente de importância. Para uma melhor compreensão da técnica, recomenda-se a seguinte literatura (SOUZA, 1988; THURSTONE (1927). Etapa 3 Determinação da matriz [ $\pi \mathrm{ij}]$ das freqüências relativas acumuladas, a partir dos resultados das freqüências das categorias ordinais calcula-se a matriz das freqüências relativas acumuladas. Etapa 4: Determinação do inverso da normal padrão das freqüências acumuladas (INPFA), a partir dos resultados obtidos na etapa anterior, calcula-se o inverso da normal padrão das freqüências acumuladas. Os resultados refletem as probabilidades de intensidade de preferências dos especialistas em relação aos estímulos (capacidades). A consecução do método partiu dos resultados da pesquisa com os especialistas, estes manifestaram suas preferências por pares de estimulos (no caso, as capacidades de inovação tecnológica), e estes submetidos as categorias ordinais $\mathrm{C}_{1}=5^{\circ}$ lugar, $\mathrm{C}_{2}=3^{\circ}$ lugar e $\mathrm{C}_{3}=4^{\circ}$ lugar). $\mathrm{O}$ resultado das preferências é, então, apresentado em ordem crescente de importância (Tabela 1).

Tabela 1 - Priorização das dimensões de capacidade de inovação tecnológica da firma em relação à performance da firma

\begin{tabular}{|c|c|c|c|c|c|c|}
\hline Dimensões (Estímulos) & C1 & C2 & C3 & $\mathbf{C 4}$ & Total & Ranking \\
\hline $\begin{array}{c}\text { Aprendizagem derivada de P \& D, das falhas e do processo de produção./ } \\
\text { capacidade da empresa para identificar forças e fraquezas internas e externas } \\
\text { oportunidades e ameaças, e para formular planos de acordo com a visão } \\
\text { corporativa e missões }\end{array}$ & $-1,22067$ & $-1,22067$ & $-1,22067$ & $-0,13971$ & $-3,8017$ & $\mathbf{1}^{\mathbf{0}}$ \\
\hline $\begin{array}{c}\text { Alocação ótima de capital/ Retorno sobre investimento } \\
\text { Intensidade de colaboração com outras empresas ou } \\
\text { centros de P \& D / Capacidade de compartilhamento de P\&D/ Taxa de sucesso } \\
\text { de P\&D de produtos/ Grau de inovação de idéias de P\&D/ Iniciativas de } \\
\text { inovação empresarial/ Porcentagem de pesquisadores empregados }\end{array}$ & $-1,22067$ & $-1,22067$ & $-1,22064$ & 0,430728 & $-3,2313$ & $3^{\circ}$ \\
\hline Capacidade para garantir a cultura organizacional da empresa & $-1,22064$ & $-0,76471$ & $-0,76471$ & 0,76471 & $-1,9854$ & $6^{\mathbf{0}}$ \\
\hline Número de patentes & $-1,22064$ & $-1,22064$ & 0,430728 & 1,220642 & $-0,7899$ & $11^{\circ}$ \\
\hline Intensidade da entrada de capital & $-1,22064$ & $-0,76471$ & 0,13971 & 0,76471 & $-1,0809$ & $\mathbf{8}^{\mathbf{o}}$ \\
\hline Tecnologia de manufatura avançada & $-1,22067$ & $-0,76471$ & $-0,43073$ & 0,430728 & $-1,9854$ & $6^{0}$ \\
\hline Previsão e avaliação de inovação tecnológica & $-1,22064$ & $-0,76471$ & 0,76471 & 0,76471 & $-0,4559$ & $12^{\circ}$ \\
\hline $\begin{array}{l}\text { Tempo de ciclo de produto/ Nível de qualidade do produto/Taxa de sucesso de } \\
\text { comercialização }\end{array}$ & $-1,22067$ & $-1,22064$ & $-0,76471$ & 0,76471 & $-2,4413$ & $4^{\circ}$ \\
\hline Capacidade de obter de fundos & $-1,22067$ & $-1,22064$ & $-0,13971$ & 1,220642 & $-1,3604$ & $7^{\mathbf{o}}$ \\
\hline Participação de mercado & $-1,22067$ & $-1,22064$ & 0,13971 & 1,220642 & $-1,081$ & $10^{\mathbf{0}}$ \\
\hline $\begin{array}{c}\text { Grau de competitividade do novo produto / Acompanhamento das forças de } \\
\text { mercado }\end{array}$ & $-1,22067$ & $-1,22067$ & $-0,43073$ & 0,430728 & $-2,4413$ & $4^{\circ}$ \\
\hline $\begin{array}{l}\text { Capacidade de gerenciar a cooperação interna e externa entre departamentos e } \\
\text { comunicação com fornecedores e clientes (Wan et al (2003) }\end{array}$ & $-1,22064$ & $-0,76471$ & 0,430728 & 1,220642 & $-0,334$ & $13^{\mathbf{o}}$ \\
\hline Unidade especializada de marketing/ Nível de qualidade pessoal/ & $-1,22067$ & $-1,22064$ & $-0,13971$ & 0,430728 & $-2,1503$ & $\mathbf{5}^{\mathbf{o}}$ \\
\hline Percentual de exportação & $-1,22067$ & $-0,43073$ & 0,13971 & 0,430728 & $-1,081$ & $9^{\circ}$ \\
\hline
\end{tabular}

Reunindo as dimensões há predominância significativa das capacidades de aprendizagem, P\&D e planejamento. Eficácia em P\&D reflete a dinâmica do processo de desenvolvimento do produto, diminui o time-to-market, melhora a rentabilidade do produto, aumenta a produtividade, entre outros benefícios. Estudo sobre a eficácia de P \& D tem várias aplicações como uma ferramenta de gestão. P\&D é forte para mensurar o desempenho semelhante ao ROI. Também pode 
ser utilizado como uma comparação (benchmark). Eficácia de P \& D também é uma medida agregada do sucesso global do produto de uma empresa no esforço de desenvolvimento. Enquanto P\&D traz consigo o percentual de pesquisadores empregados; taxa de sucesso de P\&D de produtos; número de patentes e intensidade de P\&D; a capacidade de decisão de inovação informa o grau de inovação de idéias de P\&D; a intensidade de colaboração com outras empresas ou centros de P \& D; a capacidade de compartilhamento de P\&D; a previsão e avaliação de inovação tecnológica iniciativas de inovação empresarial. Inovação tecnológica é de natureza multi-dimensional e um modelo único não pode ser suficiente para explicar o desempenho da inovação tecnológica e comportamento inovador das empresas de alta tecnologia, especialmente quando diz respeito a avaliação das atividades de inovação tecnológica sobre a organização. Por outro lado, a aprendizagem é muitas vezes utilizada para descrever o processo de inovação. É certo que as empresas inovam por meio de um constante processo de aprendizagem que geram novos conhecimentos tecnológicos (NONAKA e TAKEUCHI, 1995). Aqui as principais características do processo de inovação tecnológica são (Teece, 1986; Nelson e Winter, 1982) de natureza contínua; irreversível e afetada pela incerteza. A essência do processo de inovação tecnológica é o acúmulo de conhecimento sobre o tempo. O aumento do volume do conhecimento é produzido através de diferentes mecanismos criativos associados a diferentes modos de aprendizagem, tais como: aprendizado derivado de P \& D ou "Aprender antes de fazer" (Pisano, 1997); "Aprender fazendo", que surge espontaneamente no processo de produção (Arrow, 1962); "Aprender usando", que surge a partir dos (Rosenberg, 1982); e . "Aprender falhando", derivado da análise de decisões erradas pelos gestores de topo (MAIDIQUE e ZIRGER, 1985). Tais modos de aprendizagem, especialmente os últimos três, têm um caráter claramente progressivo na medida em que gera um fluxo contínuo de inovações tecnológicas ou novo conhecimento. Tradicionalmente, a maior importância tem sido dado a P \& D do que a outros modos de aprendizagem. E a inovação tecnológica nas empresas é um processo de aprendizagem através do qual um fluxo de novos conhecimentos. E as capacidades são geradas para as empresas mobilizar e expandir a sua tecnologia, recursos humanos e financeiros no processo de inovação. Os recursos são sempre um fator crítico para todos os tipos de atividades e processos. Evangelista et al (1997) propõem que recursos de tecnologia vão aumentar sua importância como um fator estratégico para o desempenho da empresa no futuro próximo. Alguns estudos também descobriram que a capacidade de alocação de recursos permite para sustentar a competitividade (YAM et al, 2004; GUAN e MA, 2003). 
Fase 2: Modelagem da performance global da capacidade de inovação tecnológica em relação à performance das empresas high tech

Nesta seção são avaliadas as dimensões de capacidades de inovação tecnológica em relação à performance das empresas. Este procedimento foi desenvolvido com o apoio da análise multicriterial.

Figura 2: Avaliação das dimensões da capacidade de inovação tecnológica em relação à performance da firma

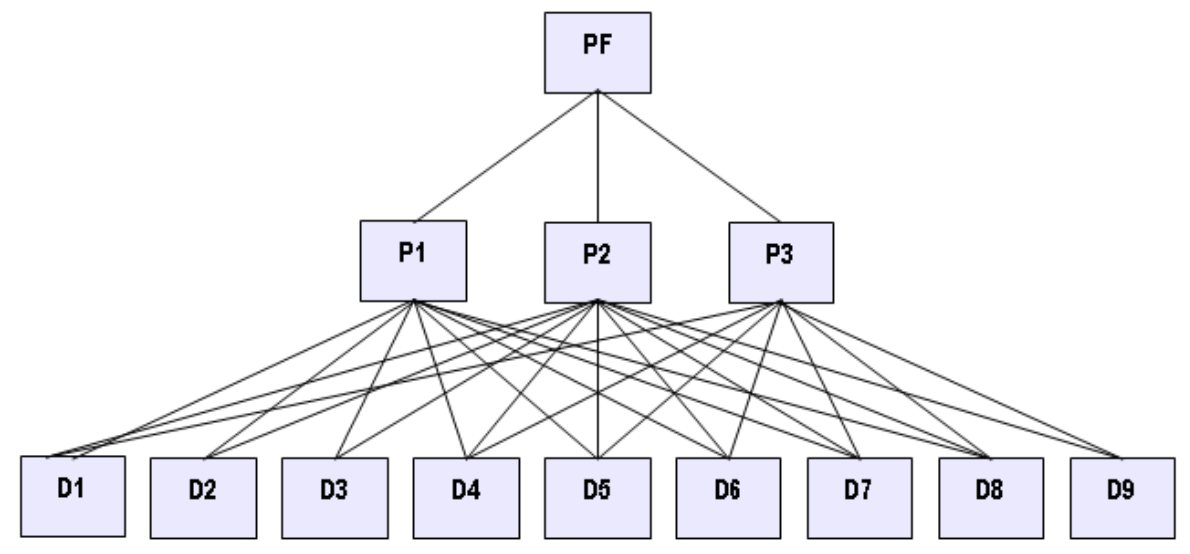

Foram utilizados os métodos Compromise Programming, Electre III e Promethee II. Os resultados alcançados confirmam a Hipótese 1: As capacidades de inovação tecnológica influenciam positivamente a performance das empresas High Tech Atribuindo-se valores para cada critério, chega-se a uma matriz de Critérios x Alternativas que fornece, juntamente com o vetor Pesos, subsídios necessários à aplicação dos métodos multicritério. Ou seja, aplica-se a metodologia de seleção e hierarquização de alternativas, utilizando-se os métodos Compromise Programming, Promethee II e Electre III.. Nesta perspectiva os métodos multicriteriais se apresentam como um instrumento viável para medir o desempenho das dimensões de capacidade de inovação em relação à performance das empresas high tech. Os resultados produzidos desta priorização permitem aos gestores concentrar melhor seus esforços e recursos na gestão das capacidades que apresentam o melhor desempenho, o que resulta no alcance dos objetivos pretendidos pelas empresas. A estrutura dessa priorização (classificação, pela análise hierárquica) é proposta em três níveis de ordenamento, numa matriz de julgamento, em que, no primeiro nível da estrutura hierárquica define o objetivo, que é alcançar a performance das empresas que vão alimentar o sistema; no segundo nível estão os critérios, que são as performances das empresas: P1: Impacto no Cliente; P2: Resultado dos Negócios; P3: Percentual de Vendas de Produtos Inovadores; ; no terceiro nível estão as alternativas, que são as dimensões capacidades de inovação tecnológica: D1: Capacidade de P\&D (GUAN e MA, 2003; BURGELMAN et al, 2004; YAM et al, 2004); D2: Capacidade de Decisão de Inovação (BARTON, 1984); D3: Capacidade de Marketing (ACHILLADELIS, e ANTONAJIS, 2001; GUAN e MA, 2003; YAM et al, 2004; KIM et al, 2005); D4: Capacidade de Manufatura 
(GUAN e MA, 2003; YAM et al, 2004); D5: Capacidade de Capital (YAM et al, 2004); D6: Capacidade de Alocação de Recursos (LAU, YAN e TANG, 2010); D7: Capacidade de Planejamento Estratégico (LAU, YAN e TANG, 2010); D8: Capacidade de Aprendizagem (LAU, YAN e TANG, 2010); D9: Capacidade Organizacional (LAU, YAN e TANG, 2010). O processo de priorização obedece ao julgamento dos avaliadores (especialistas). De posse dos resultados da matriz de julgamento, aplicou-se os métodos: Promethee II, Electre III e Compromise Programming para avaliar as capacidades de inovação em relação à performance das empresas. Os resultados produzidos são referenciados na Tabela 2.

Tabela 2: Avaliação das preferências - Capacidade de inovação tecnológica x Performance de empresa high tech

\begin{tabular}{cccc}
\hline Dimensões das Capacidades de Inovação Tecnológica & \multicolumn{2}{c}{ Classificação } \\
Capacidade de P\&D / Aprendizagem & Promethee II & Compromise Programming & Electre III \\
Capacidade Planejamento estratégico & $1^{\mathrm{a}}$ & $1^{\mathrm{a}}$ & $2^{\mathrm{a}}$ \\
Capacidade de alocação de recursos / capacidade & $2^{\mathrm{a}}$ & $3^{\mathrm{a}}$ & $3^{\mathrm{a}}$ \\
organizacional / Capacidade de capital & $3^{\mathrm{a}}$ & $4^{\mathrm{a}}$ & $2^{\mathrm{a}}$ \\
Capacidade de Marketing & $4^{\mathrm{a}}$ & $4^{\mathrm{a}}$ & $3^{\mathrm{a}}$ \\
Capacidade de Manufatura & $4^{\mathrm{a}}$ & & \\
\hline
\end{tabular}

Os resultados produzidos pelos métodos evidenciam as capacidades de P\&D e Aprendizagem como as mais significativas para assegurar a performance da empresa. Ao comparar os resultados em termos de desempenho, os métodos Compromise Programinng e Promethee II não apresentaram diferenças em suas classificações. Já em relação ao Electre III, os resultados foram divergentes. E isto deve-se aos limiares de veto $\mathrm{p}, \mathrm{q}$ e $\mathrm{v}$, respectivamente, de indiferença, preferência forte e veto ou incomparabilidade, há uma discrepância na estrutura de seus resultados (classificação). O Electre III apresenta um grupo de solução com estrutura hierárquica mais flexível. Isto se deve a própria concepção do método, como também à consideração bastante explícita do aspecto da indiferença e incomparabilidade entre alternativas. Os resultados referenciados pelos métodos Promethee II e Compromise Programming refletem a preferência, na visão dos especialistas pelas capacidades de inovação tecnológica em P\&D e Aprendizagem. A essência do processo da inovação tecnológica é o acúmulo de conhecimento sobre tempo. O aumento do volume do conhecimento é produzido por meio de diferentes mecanismos associados com os diferentes modos de aprendizagem, tais como: aprendizado derivado de P \& D ou Aprender antes de fazer (Pisano, 1997); Aprender fazendo, que surge espontaneamente no processo de produção (Arrow, 1962); Aprender usando, que surge a partir de observação de diferentes formas em que os clientes usam os produtos da empresa (Rosenberg, 1982); e Aprender falhando, derivado da análise de decisões erradas pelos gestores de topo (MAIDIQUE e ZIRGER, 1985). Esses modos de aprendizagem, especialmente os últimos três, têm um caráter claramente progressivo na medida em que geram um fluxo contínuo de inovações tecnológicas. Mas é certo tradicionalmente a maior importância é para P \& D do que a outros modos de aprendizagem (NIETO, 2004). À luz da 
literatura especializada (Evangelista et al, 1997), P\&D tem forte impacto na performance da empresa. P \& D é um componente central das atividades de inovação tecnológica das empresas (EVANGELISTA et al, 1997). Na verdade, muitos estudos sobre inovação empregam P \& D como indicadores de inovação tecnológica. P \& D é considerada uma peça-chave das atividades de inovação.

\section{Fase 3: Determinação da correlação da capacidade de inovação tecnológica das empresas}

Nesta seção são determinadas as correlações entre as capacidades de inovação tecnológica das empresas. A correlação de Spearman é freqüentemente usada para descrever a relação entre duas características ordinais. Os dados foram extraídos de especialistas por meio de uma matriz de julgamento. Os resultados são apresentados na Tabela 3.

Tabela 3: Correlação entre as dimensões de capacidades de inovação tecnológica da firma

\begin{tabular}{|c|c|c|c|c|c|c|c|c|c|}
\hline \multirow{2}{*}{$\begin{array}{l}\text { Variáveis: Capacidades de } \\
\text { Inovação Tecnológica da Firma }\end{array}$} & \multicolumn{9}{|c|}{ Performance de Capacidade de Inovação Tecnológica da Firma } \\
\hline & 1 & 2 & 3 & 4 & 5 & 6 & 7 & 8 & 9 \\
\hline D1: Capacidade de P\&D & 1 & & & & & & & & \\
\hline D2: Capacidade de Decisão de Inovação & $\mathbf{0 , 8 1}$ & 1,00 & & & & & & & \\
\hline D3: Capacidade de Marketing & 0,72 & 0,92 & 1,00 & & & & & & \\
\hline D4: Capacidade de Manufatura & 0,85 & 0,50 & 0,52 & 1,00 & & & & & \\
\hline D5: Capacidade de Capital & 0,74 & 0,55 & 0,46 & 0,59 & 1,00 & & & & \\
\hline D6: Capacidade de Alocação de Recursos & 0,25 & 0,27 & 0,14 & 0,15 & 0,70 & 1,00 & & & \\
\hline D7: Capacidade de Planejamento Estratégico & 0,13 & $(\mathbf{0 , 0 2})$ & $(\mathbf{0 , 0 6})$ & 0,32 & $(0,17)$ & $(\mathbf{0 , 2 4})$ & 1,00 & & \\
\hline D8: Capacidade de Aprendizagem & 0,47 & 0,30 & 0,33 & 0,50 & 0,53 & 0,08 & 0,40 & 1,00 & \\
\hline D9: Capacidade Organizacional & 0,45 & 0,35 & 0,06 & 0,16 & 0,62 & 0,35 & $(\mathbf{0 , 1 0})$ & 0,32 & 1,00 \\
\hline
\end{tabular}

Reunindo as dimensões de capacidades há forte correlação entre as capacidades de decisão de inovação, P\&D e os esforços de marketing. É certo também que a capacidade para planejar não tem correlação com as demais capacidades. Planejar é a capacidade da empresa para identificar forças e fraquezas internas e externas, oportunidades e ameaças, e para formular planos de acordo com a visão corporativa e missão. Os resultados alcançados estão alinhados com o estado da arte que aponta forte correlação entre P\&D e marketing e decisão de inovação.

\section{Fase 4: Modelagem da taxa ótima de performance de capacidade de inovação tecnológica da empresa}

Esta fase centra-se na determinação da taxa de efetividade òtima da capacidade de inovação tecnológica das empresas high tech utilizando a modelagem neurofuzzy. É um processo cujos atributos em sua maioria possuem características de elevada subjetividade, em que a experiência do tomador de decisão é bastante significativa. Neste espectro há uma necessidade de uma ferramenta que permite a agregação das variáveis quantitativas e qualitativas que convergem rumo a um único 
parâmetro de avaliação (CURY e OLIVEIRA; 1999; VON ALTROCK, 1997). Este modelo agrega a tecnologia de Redes Neurais e Lógica Fuzzy (tecnologia neurofuzzy). Aqui este modelo suporta o planejamento da capacidade de inovação tecnológica das empresas high tech, à medida que permite avaliar a melhor taxa de capacidade para o alcance da performance das empresas high tec. $\mathrm{O}$ modelo aqui apresentado tem como referência o modelo de Cury e Oliveira (1999). À luz da tecnologia neurofuzzy os dados de entrada de natureza qualitativa são agrupados para determinar os parâmetros de comparação entre as alternativas. A técnica está estruturada a partir de uma combinação de todos os atributos em blocos de inferência que usam regras de base fuzzy e expressões linguísticas, de modo que a preferência para cada alternativa de decisão prioridade dos determinantes da taxa ótima de performance capacidade de inovação tecnológica, em termos de benefícios para a empresa, pode ser expressa por meio de um intervalo, variando de 0 a 10 . A modelagem é composta de variáveis qualitativas e quantitativas, com base em informações dos especialistas. Descreve-se a seguir o modelo neurofuzzy.

Determinação das variáveis de entradas: Esta seção centra-se na determinação das variáveis de entrada (VE) do tipo qualitativas e quantitativas. Essas variáveis foram extraídas das variáveis independentes (dimensões de capacidade de inovação tecnológica das empresas). Os termos lingüísticos atribuídos a cada VE apresentadas são: Alto, Médio e Baixo. Assim, a VE's mostradas no modelo são apresentadas na Tabela 1, as quais são transformadas em variáveis lingüísticas, com seus respectivos Graus de Convicção ou de Certeza (GdC), com a intervenção de vinte juízes opinando no processo. Os graus atribuídos pelos juízes são convertidos em expressões lingüísticas, com seus respectivos $\mathrm{GdC}$, com base nos conjuntos fuzzy e nas regras SE (agregação das regras) - ENTÃO (composição das regras). 


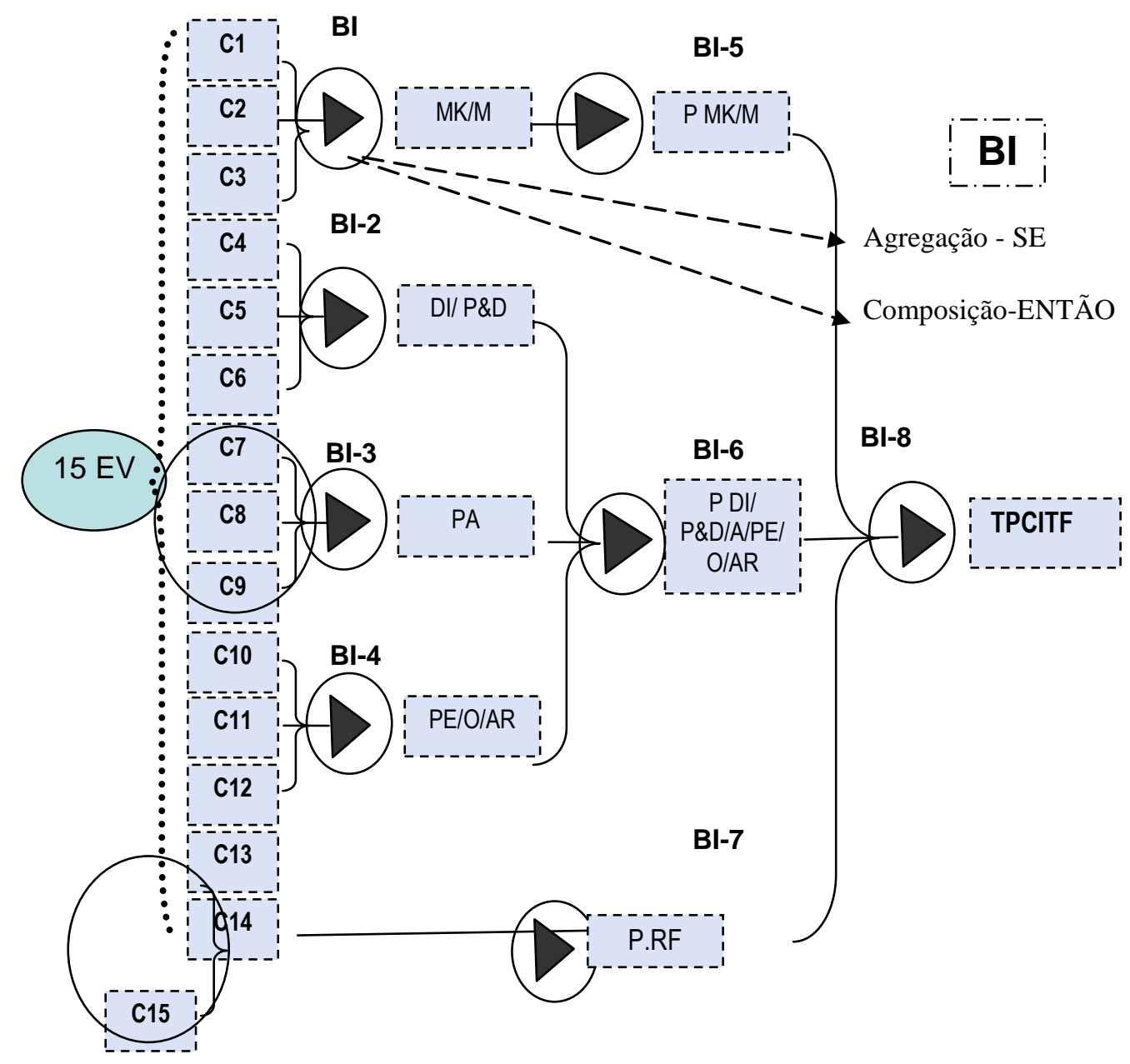

Determinação das variáveis intermediárias e termos linguísticos: As variáveis de entrada de natureza qualitativa passam pelo processo de inferência fuzzy, resultando em termos lingüísticos de variáveis intermediárias (VI). Assim, os termos lingüísticos atribuídos às VI são: Baixo, Médio e Alto. As variáveis intermediárias foram obtidas em: Desempenho Marketing e Manufatura; Desempenho de Decisão de Inovação e P\&D; Desempenho de Aprendizagem; Desempenho de Planejamento Estratégico, Organização e Alocação de Recursos. A Configuração Performance Marketing e Manufatura; Performance Decisão de Inovação, P\&D, Planejamento Estratégico, Organização e Alocação de Recursos; Performance Retorno Financeiro. A arquitetura proposta é composta de oito configurações de sistemas especialistas fuzzy, quatro variáveis de entrada (VE) de natureza qualitativa que passam pelo processo fuzzy e através do bloco de inferência, portanto, produzindo uma variável de saída (VS), denominada variável intermediária (VI). Por sua vez, VIs, que se juntam a outras variáveis VIs, formando portanto, um conjunto de novas VEs, conseqüentemente configurando uma sequência até a última camada da rede. Na última camada da 
rede é definida a variável de saída (VS) da Rede neurofuzzy. Esta VS então é submetida a um processo defuzzificação para alcançar o resultado final: Taxa de Efetividade Ótima de Performance de Capacidade de Inovação Tecnológica de Empresas High Tech. Em síntese, a inferência fuzzy ocorre a partir da base de regras, gerando o vetor lingüístico da VS, obtido por meio das etapas de agregação e composição. A título de exemplo, ao solicitar a opinião de um dos especialistas sobre qual a taxa de efetividade ótima de performance de capacidade de inovação tecnológica da empresa A, a resposta foi 8,0. Em seguida, realizou-se o processo de fuzzificação (simulação), atribuindo termos lingüísticos BAIXA, MÉDIA e ALTA a graus de avaliação em uma escala de 1 a 10. Para o grau 8, considerado BAIXA por $0 \%$ dos especialistas, MÉDIA por $55 \%$ e ALTA por $45 \%$ dos especialistas. Em síntese, com as respostas dos especialistas foi possível determinar os graus de certeza dos termos lingüísticos de cada uma das variáveis de entrada, por meio da utilização dos conjuntos fuzzy. Os resultados confirmam a hipótese 2: A interação entre as dimensões de capacidades de inovação tecnológica relaciona-se com a taxa de efetividade ótima da performance das empresas High Tech. Foram definidos os conjuntos fuzzy genéricos para todas as VEs qualitativas, que apresentam sempre três níveis de termos lingüísticos: um inferior, um médio e outro superior. Após a conversão de todas as VEs em suas correspondentes variáveis lingüísticas, com seus respectivos GdC, os blocos de inferência fuzzy (BI), compostos por base de regras SEENTÃO, são operados com base nos operadores MIN-MAX, obtendo-se um valor lingüístico para cada variável intermediária e para variável de saída do modelo, com os termos lingüísticos definidos pelos juízes, anteriormente. A partir das variáveis de entradas (características extraídas dos projetos de desenvolvimento de produtos), geram-se a base de regras. Cada regra possui um fator de ponderação individual, denominado de Fator de Certeza (FdC), compreendido entre 0 e 1 , que indica o grau de importância de cada regra na base de regras fuzzy. E a inferência fuzzy ocorre a partir da base de regras, gerando o vetor lingüístico da VS, obtido por meio das etapas de agregação e composição.

Determinação da variável de saída - taxa de efetividade ótima da capacidade de inovação tecnológica: A variável de saída (VS) do modelo neurofuzzy proposto foi denominada de Taxa de Efetividade Òtima de performance de inovação tecnológica de empresas high tech. O processo de fuzzificação envolve a determinação das funções de pertinência para cada uma das variáveis de entrada. Se os dados de entrada forem valores precisos, resultados de medições ou observações, é necessário efetuar-se a estruturação de conjuntos fuzzy para as variáveis de entrada, consistindo no processo de fuzzificação. Caso as variáveis de entrada sejam obtidas em valores lingüísticos, não é necessário o processo de fuzzificação. Defuzificação: No caso de aplicações que envolvem variáveis qualitativas, como é o caso em questão, é necessário um valor numérico como resultado do sistema, denominado de defuzzificação. Sendo assim, após a inferência fuzzy é 
necessário a fuzzyficação, ou seja, transformar os valores lingüísticos em valores numéricos, a partir de suas funções de pertinência (VON ALTROCK, 1997). O método do Centro de Máximos se popularizou para a determinação de um valor exato para o vetor lingüístico da VS. A partir desse método, definem-se os graus de certeza dos termos lingüísticos como "pesos" associados a cada um destes valores. O valor de compromisso exato (VC) é determinado através da ponderação dos pesos com relação aos valores típicos (valores máximos das funções de pertinência), conforme Equação apresentada a seguir (VON ALTROCK, 1997; CURY e OLIVEIRA, 1999).

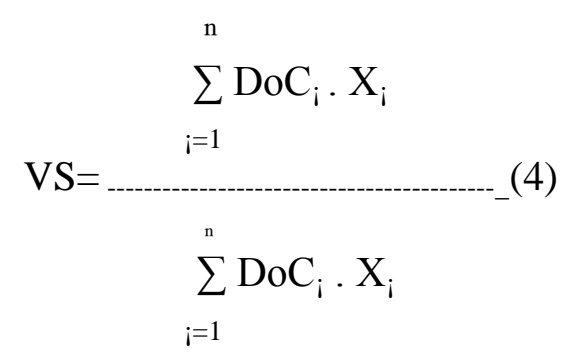

Onde i GdC representam os graus de certeza dos termos lingüísticos da variável de saída final e i X indica os valores típicos para os termos lingüísticos, que correspondem aos máximos dos conjuntos fuzzy que definem a variável de saída final. A título de demonstração, utilizando-se graus atribuídos (média) hipotéticos, entra-se na expressão do cálculo do TPCITj com os GdCi do seguinte vetor lingüístico da variável de saída (TODSP), também hipotético: BAIXO=0,30; MÉDIO=0,49; $\mathrm{ALTO}=0,14$. $\mathrm{O}$ valor do TPCITF numérico numa escala de 0 a 1 corresponde a 0,7352 , resultante da média aritmética dos valores resultantes da defuzzificação de cada um dos vinte juízes simulados. Este valor corresponde a um valor médio para o TEPC. Com esse resultado apresentado pela taxa de efetividade ótima de performance de capacidade de inovação tecnológica é possível dizer que a taxa ótima para o projeto A (hipotético) do projeto deve ser pelo menos 0,7352 . Sem dúvida é mais um instrumento nas mãos dos gestores de gestão de inovação tecnológica em empresas high tech.

\section{Palavras finais: lições aprendidas}

Este estudo teve por objetivo contribuir para uma política de planejamento de inovação em empresas high tech no Brasil. Para isto apresenta uma metodologia para avaliar a capacidade de inovação tecnológica na performance desta categoria de empresas. O estudo procurou cobrir um espaço existente na literatura sobre planejamento de Inovação na perspectiva da capacidade de inovação tecnológica. Para isto concebe um conjunto de métodos de escalagem psicométrica, análise multicriterial e inteligência artificial, num contexto de incerteza e subjetividade. Esse procedimento permite reduzir a subjetividade nos resultados alcançados. Presume-se como pressuposto mais forte o fato de reconhecer a importância da subjetividade no julgamento dos 
decisores; seus valores, seus objetivos, seus preconceitos, sua cultura, sua intuição, assim como a influência dos fatores subjetivos sobre a percepção e entendimento das variáveis envolvidas.

Aqui a proposta de modelagem apresentada ganha ênfase, essa diversidade de métodos quando combinada são instrumentos valiosos e de elevado potencial e valor agregado de grande envergadura, contribuindo para a robustez da modelagem. A factibilidadade da tecnologia neurofuzzy, sobretudo na interação de variáveis qualitativas e quantitativas utilizada na modelagem do processo, mostrando-se determinante no levantamento da taxa de efetividade ótima da performance da capacidade de inovação tecnológica à luz de empresas high tec. Por se tratar de um procedimento no qual os atributos possuem características de subjetividade, com a intervenção de especialistas, a tecnologia neurofuzzy foi determinante e significativa na classificação das variáveis qualitativas e quantitativas convergidas a um único parâmetro de avaliação, a taxa de efetividade ótima de performance de inovação tecnológica. Isto favorece as tomadas de decisões num contexto de incerteza. Esta proposta é mais um instrumento nas mãos dos gestores, com grande efeito na redução da incerteza gerada em decisões de inovação tecnológica. Evidentemente permanecem diversas questões a serem aprofundadas em outros estudos do gênero e espera-se ter contribuído para uma discussão metodológica plausível, mas que ainda possa ser bastante explorada. É certo que a Inovação coloca desafios significativos tanto para pesquisa como para a prática, impõe a necessidade de uma aprendizagem ativa nas empresas high tech. Esse aprendizado envolve não só o desenvolvimento de novas capacidades dentro da empresa, mas transpõe fronteiras. Interações com outras empresas, outros conhecimentos e especialistas estão se tornando um foco importante e emergente para a inovação tecnológica.

Das diversas dimensões, os resultados referenciam predominância nos esforços de P\&D, mas é certo que essas capacidades de inovação estão na ordem do dia e devem ser balizadas como uma prioridade do momento, no contexto dos esforços sistêmicos orientados pela definição e redefinição de performance das empresas high tech ao longo do tempo. É plausível que a construção das capacidades se dão ao longo de um processo contínuo e converge à performance desejada, que está em constante transformação pela aquisição de novas demandas. Por esta via, a política de inovação nesta categoria de empresas haverá de estar ancorada em um planejamento eficiente. Espera-se que as capacidades de P\&D possam abrir espaços permitindo as empresas high tech expandir suas tecnologias existentes e estabelecer novas tecnologias ou melhorar a função de P\&D. As capacidade de P\&D compreendem principalmente um número significativo de pesquisadores empregados (LEFEBVRE et al, 1998), a taxa de sucesso de P \& D de produtos autogerada por produtos inovadores, número de patentes (ACHILLADELIS e ANTONAJIS, 2001; DAMANPOUR e WISCHNEVSKY, 2006), e intensidade de P \& D (ACHILLADELIS e ANTONAJIS, 2001; YAM et al, 2004; DAMANPOUR e WISCHNEVSKY, 2006). 
Esses critérios são medidos quantitativamente e qualitativamente. Já as capacidades de inovação de decisão remetem a capacidade de executar as decisões de inovação tecnológica na empresa. Estas habilidades incluem o grau de inovação da P \& D, a intensidade da colaboração com outras empresas ou centros de P \& D (LEFEBVRE et al, 1998; ACHILLADELIS e ANTONAJIS, 2001), a capacidade de P\&D partilhar conhecimento (GUAN e MA, 2003), previsão e avaliação de inovação tecnológica (YAM et al, 2004; BURGELMAN et al., 2004), e iniciativas de inovação empresarial (GUAN e MA, 2003). Essas habilidades são avaliadas subjetivamente. Recursos de marketing significam uma firme capacidade para promover e vender produtos com base na demanda, que é primariamente influenciado pelo mercado (MANU e SRIRAM, 1996), grau de competitividade do novo produto, monitoramento das forças de mercado (GUAN e MA, 2003), unidade especializada de marketing, (ACHILLADELIS e ANTONAJIS, 2001), e as percentagens de exportação (GUAN e MA, 2003). Todas essas variáveis são de natureza subjetiva. Num segundo momento, os esforços são para as capacidades de produção, em que as empresas devem transformar P \& D em resultados técnicos de produtos e melhorias na qualidade do produto. Capacidade de manufatura, tais como tecnologia de fabricação avançada (GUAN e MA, 2003), o nível de qualidade do produto, taxa de sucesso de comercialização (YAM et al, 2004), nível de qualidade do pessoal da produção (YAM et al, 2004) e o tempo de ciclo do produto (GUAN e MA, 2003) são avaliados subjetivamente. Por fim, capacidades de capitais que compreendem as condições necessárias para garantir que o avanço das capacidades tecnológicas das empresas são provenientes principalmente das capacidades de captação de recursos, alocação ideal de entrada de capital (BURGELMAN et al, 2004), da intensidade de capital (GUAN e MA, 2003) e o retorno sobre o investimento (MANU e SRIRAM, 1996). Claro está também, que a listagem de prioridades de capacidades de inovação tecnológica é dinâmica, depende das capacidades existentes, essenciais e desejadas, que vão surgindo ao longo da prática, sempre colocando em pauta novos conceitos, novos conteúdos e demandando novos comportamentos e implementos técnicos, o que requer fundamentalmente e igualmente a reconfiguração permanente e recorrente que entra na pauta de novas capacidades de inovação à luz de novas performances. Ainda neste esforço, as pesquisas dessas prioridades hão de ser aplicadas de forma permanente e recorrente.

\section{Abstract}

The present paper aims to contribute to the planning guidelines in the field of innovation in firms high tech in Brazil. Therefore, a methodological support for Evaluating technological innovation capability in performance of the firms. Thus, it develops a methodological support for evaluation the technological innovation capabilities that considers a sequence of systematic procedures in the following phases: Phase 1: determination of the conceptual model; and phase 2: Verification of the conceptual model. Several support instruments were used psychometric scales; multi-criteria; Neuro-fuzzys..The results produced are satisfactory, validating the proposed procedure for 
Evaluating firm technological innovation capability. In this scenario, our methodological contribution is highlighted, because it provides support to the critical priorities in order to implement this project, and is also directed to building up innovation as a key element for product development. We look forward to a more practical and efficient orientation that supports its longterm goals, thus assuring national competitiveness concerning the category of priorities. By gathering the cognitive elements, it can be seen that this strategy requires a priority dynamics, which depends on the initial state of product development process, on the concrete characteristics of the projects and on an innovation policy.

Key-words: methodology; evaluation; technological innovation capability; performance of the firms high tech.

\section{Referências}

ACHILLADELIS, B.; ANTONAJIS, N. The dynamics of technological innovation: the case of the pharmaceutical industry. Research Policy, v. 30, p. 535-588, 2001. crossref

ADLER, P. S.; SHENBAR, A. Adapting your technological base: the organizational challenge. Sloan Management Review, v. 25, p. 25-37, 1990.

AFUAH, A. Innovation Management: Strategies, Implementation, and Profits. New York: Oxford University Press, 1998.

ARROW, KENNETH J. The economic implications of learning by doing. The Review of Economic Studies, v. 29, n. 3 , p. 155-173, 1962. crossref

BELLMAN, R.; ZADEH, L.A. Decision-making in a fuzzy environment. Management Science v. 17, p. 141-164, 1970. cross ref

BROWN, S.L., K.M. EISENHARDT. Product development: Past research, present findings, and future directions. Acad. Management Rev, v. 20, p. 343-378, 1995.

CALANTON, R.; VICKERY, S.; DEOGE, C. Business Performance and Strategic New Product Development Activities: An Empirical Investigation. Journal of Product Innovation Management, v. 12, n. 3, p. 214-223, 1995. crossref

CHESBROUGH, H.; ROSENBLOOM, R.S. The role of the business model in capturing value from innovation: Evidence from Xerox Corporation's technology spin-off companies. Industrial and Corporate Change, v. 11, n. 3, p. 529-555, 2002. crossref

CHIESA, V.; COUGHLAN, P.; VOSS, C.A. Development of a technical innovation audit. Journal of Product Innovation Management, v. 13 n. 2, p. 105-36, 1996. crossref

CHRISTENSEN, C.M. The Innovator's Dilemma. Harper Business Essentials, New York, NJ, 2003.

CURY, M. V. Q. Modelo Heurístico Neurofuzzy para Avaliação Humanística de Projetos de Transporte Urbano. Tese submitted for the degree of. Doctoral of Science in Production Engineering of University Federal of Rio de Janeiro, COPPE/UFRJ., 1999.

DAMANPOUR, F. Organizational complexity and innovation: developing and testing multiple contingency models. Management Science, v. 42, n.5, p. 693-716, 1996. cross ref

EVANGELISTA, R.; PERANI, G.; RAPITI, F. E; ARCHIBUGI, D. Nature and impact of innovation in manufacturing: some evidence from the Italian innovation survey. Research Policy, v. 26, p. 521-36, 1997. crossref

GARCI'A-MUIN ${ }^{\sim}$ A, F.E.; NAVAS-LO’ PEZ, J.E. Explaining and measuring success in new business: the effect of technological capabilities on firm results. Technovation, v. 27, n.1-2, p. 30-46, 2007.

GOICOECHEA, A.; HANSEN, D.R. E; DUCKSTEIN, L. Multiobjective Decision Analysis with Engineering and Business Applications - John Wiley \& sons, inc., Chichester, Inglaterra, 1982. 
GRILICHES, ZVI. Patent Statistics as Economic Indicators: A Survey. Journal of Economic Literature, v. 28, p.1661-1707, 1990.

GUAN, J., MA, N. Innovative capability and export performance of Chinese Firms. Technovation, v. 23, n. 9, p. 737747, 2003. cross ref

GUAN, J.C.; YAM, R.C.M.; MOK, C.K.; MA, N. A study of the relationship between competitiveness and technological innovation capability based on DEA models. European Journal of Operational Research, v. 170, p. 971-986, 2006. crossref

KIM, J.Y.; WONG, V.; ENG, T.K. Product variety strategy for improving new product development proficiencies. Technovation, v. 25, n. 9, p. 1001-1015, 2005. crossref

LALL, S. Technological capabilities and industrialization. World Development, v. 20, p. 165-186, 1992. crossref

LAU, A.K.W.; YAM, R.C.M.; TANG, E.P.Y, The impact of technological innovation capabilities on innovation performance An empirical study in Hong Kong. Journal of Science and Technology Policy in China, v. 1, n. 2, p. 163-186, 2010. crossref

MADIQUE, M.A.; ZIRGER, B.J. A study of success and failure in product innovation: The case of the U.S. electronics industry. IEEE Transactions on Engineering Management, v. 31, n.4, p. 192-203, 1984. cross ref

NELSON, R.; WINTER, S. An evolutionary theory of economic change. Cambridge, Mass.: Harvard University Press, 1982.

NIETO, M. Basic propositions for the study of the technological innovation process in the firm, European Journal of Innovation Management, v. 7, n. 4, p.314 - 324, 2004. crossref

NONAKA, I.; TAKEUCHI, H. The Knowledge-Creating Company, Oxford University Press, New York, NY, 1995.

PISANO, G. Knowledge, integration, and the locus of learning: an empirical analysis of process development.

Strategic Management Journal, v. 15, p. 85-100, 1994. crossref

PRAHALAD, C. K.; HAMEL, G. The core competence of the corporation. Harvard Business Review, p. 79-91, May/June, 1990.

ROSENBERG, N. Inside the Black Box: Technology and Economics Cambridge, 1982.

SOUZA, J. 1988. Métodos de Escalagem Psicossocial. Vol. V, Brasília: Thesaurus.

TEECE, D.J.; PISANO, G.; SHUAN, A. Dynamic capabilities and strategic management. Strategic Management Journal, v. 18, n.7, p.509-33, 1997. crossref

TEECE, DAVID J. Profiting from technological innovation: Implications for integration, collaboration, licensing and public policy. Research Policy, v.15, p. 285-305, 1986. cross ref

TUSHMAN, M. L.; NADLER, D. A. Organizing for innovation. California Management Review, v. 28 . n.3, p. 74. University Press, Cambridge, 1986.

VON ALTROCK, C. Fuzzy Logic and Neurofuzzy: Applications in Business and Finance. Prentice Hall, USA, 1997.

WANG, C.; LUB, I.; CHENC, C. Evaluating firm technological innovation capability under uncertainty.

Technovation, v. 28, p. 349-363, 2008. crossref

WHEELWRIGHT, S.; CLARK, K. Revolutionising. Product Development. Free Press, New York, $1992 .$.

YAM, et.al. An audit of technological innovation capabilities in Chinese firms: some empirical findings in Beijing, China. Research Policy, v. 33, n.8, p. 1123-1140, 2004. crossref

ZADEH, L.A. Fuzzy sets. Information and Control, v. 8, p. 338-353, 1965. crossref 


\section{Dados dos autores}

Nome completo: Selma Regina Martins Oliveira

Filiação institucional: Universidade de São Paulo

Departamento: Administração

Função ou cargo ocupado: Professora colaboradora

e-mail: selmaregina@webmail.uft.edu.br

Nome completo: Jorge Lino Alves

Filiação institucional: Universidade do Porto

Departamento: INEGI, Faculdade de Engenharia

Função ou cargo ocupado: Professor Associado

e-mail: falves@fe.up.pt

Submetido em: 23/09/2012

Aceito em: 14/12/2013 